\title{
Comprehensive Molecular Genetic Diagnostics of Birt-Hogg-Dube Syndrome in a Russian Patient with Renal Cancer and Lung Cysts: A Case Report
}

\author{
Dmitry S. Mikhaylenko a, b Vsevolod B. Matveev ${ }^{c} \quad$ Margarita G. Filippova ${ }^{c}$ \\ Kirill I. Anoshkin ${ }^{a}$ Nikolay A. Kozlov ${ }^{c}$ Alexander V. Khachaturyan ${ }^{c}$ \\ Alexandra V. Semyanikhinac Sergey D. Nifatov ${ }^{c}$ Alexander S. Tanas ${ }^{a}$ \\ Marina V. Nemtsovab Dmitry V. Zaletayev ${ }^{a}$, b \\ aDepartment of Oncogenetics, Research Centre for Medical Genetics, Moscow, Russia; \\ bLaboratory of Medical Genetics, Institute of Molecular Medicine, I.M. Sechenov First \\ Moscow State Medical University (Sechenov University), Moscow, Russia; 'Department of \\ Oncological Urology, N.N. Blokhin National Medical Research Center of Oncology, Moscow, \\ Russia
}

\section{Keywords}

Birt-Hogg-Dube syndrome · Case report · DNA sequencing $\cdot$ Renal cancer · Lung cyst

\begin{abstract}
We report a case of Birt-Hogg-Dube syndrome (BHDS), a rare hereditary syndrome, the main visible sign of which is the development of multiple skin fibrofolliculomas. In our case, there was a manifestation of BHDS consisting in the absence of fibrofolliculomas and presence of other characteristic features of this syndrome: lung cysts and renal cancer. The 26 -year-old woman was admitted to a clinic for diagnosis and treatment of a neoplasm of the left kidney and had a history of renal cell cancer (RCC) of the right kidney and spontaneous pneumothorax. Multiple tumors of the left kidney and lung cysts were observed upon clinical and laboratory testing. Tumors of the left kidney were resected and diagnosed by a pathologist as chromophobe RCC. Sequencing of FLCN exons 4-14 from blood DNA revealed the heterozygous germline nonsense mutation c.1429C>T (p.R477*), confirming the diagnosis of BHDS. Several somatic variants were detected by tumor DNA sequencing using the Comprehensive Cancer Panel and lon S5 platform. Medical-genetic counseling was conducted, and follow-up
\end{abstract}


management was outlined. To our knowledge, this case report is the first comprehensive clinical and genetic examination of a patient with BHDS in Russia. The p.R477* mutation has been described by other authors in patients with fibrofolliculomas and lung cysts, but not in those with RCC, while RCC was the first manifestation of BHDS in our case. The case report may help geneticists, oncologists, and other specialists to better understand the clinical and genetic heterogeneity of BHDS in various populations.

(C) 2021 The Author(s).

Published by S. Karger AG, Basel

\section{Introduction}

Hereditary cancer syndromes account for $3 \%$ of all renal cell cancer (RCC) cases. The set includes monogenic disorders, such as Von Hippel-Lindau syndrome, Birt-Hogg-Dube syndrome (BHDS), hereditary leiomyomatosis and RCC, hereditary papillary RCC type 1 , and $B A P 1$ tumor predisposition syndrome. Direct DNA diagnosis and detection of a pathological mutation in a single candidate gene make it possible to ultimately diagnose the disorder, identify the target organs most susceptible to carcinogenesis based on known genotypephenotype correlations in some cases, and adjust the treatment and follow-up tactics depending on the higher lifetime risk of certain cancers and presence or absence of specific targets for targeted drugs [1,2].

BHDS (OMIM 135150) is a rare autosomal dominant cancer syndrome $(1: 200,000)$ with multiple fibrofolliculomas and, sometimes, trichodiscomas being the main manifestations in $90 \%$ of cases. The tumors are located predominantly on the face, neck, and upper trunk. Lung cysts develop in approximately $80 \%$ of patients with BHDS and affect mostly the basal segments of the lungs. Cysts increase in number and diameter with time and lead to spontaneous pneumothorax in $30 \%$ of cases, threatening the patient's life. Kidney tumors develop in $50 \%$ of patients with BHDS [3].

Germline mutations of the FLCN tumor suppressor gene are responsible for BHDS. FLCN is in chromosome 17p11.2 and codes for folliculin. Relevant mutations are loss-of-function mutations and include small deletions/insertions, complex frameshifts, nonsense mutations, and splicing site mutations; missense mutations are far less commonly identified. The C8 mononucleotide tract in exon 11 is a hotspot of FLCN. Single-nucleotide deletions or insertions in the tract account for approximately 50\% of BHDS cases. Folliculin forms a complex with FNIP1/2 and is involved in regulating the mTOR pathway (circumstance possibly explaining the high proportion of hybrid kidney tumors with the oncocytic component), $5^{\prime}$-AMP-activated protein kinase, TFE3/TFEB transcriptional activity, and other processes in the cell $[4,5]$.

The minimal diagnostic criteria used to presumptively diagnose BHDS include the following: (1) at least 2 characteristic skin neoplasms, of which at least one has histologically been identified as fibrofolliculoma after puberty; (2) bilateral (multifocal) cysts found in the basal segments of the lungs before 40 years of age and family history of the disease; (3) bilateral (multifocal) chromophobe or hybrid oncocytic-chromophobe tumors of the kidneys in hereditary RCC; and (4) a combination of skin, pulmonary, and renal BHDS symptoms in the patient and pedigree [6]. RCC is often multifocal and bilateral in BHDS and may belong to various pathomorphological types, including chromophobe carcinoma, hybrid oncocyticchromophobe tumors, and, less commonly, clear cell, and papillary carcinomas [7]. BHDS is highly heterogeneous clinically and therefore difficult to diagnose based on clinical findings alone, warranting molecular genetic testing.

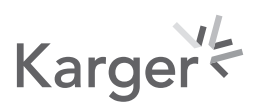




\section{Case Reports in Oncology}

\begin{tabular}{l|l}
\hline Case Rep Oncol 2021;14:963-971 \\
\hline DOI: 10.1159/000516763 & $\begin{array}{l}\text { @ 2021 The Author(s). Published by S. Karger AG, Basel } \\
\text { www.karger.com/cro }\end{array}$ \\
\hline
\end{tabular}

Mikhaylenko et al.: Case of Birt-Hogg-Dube Syndrome with the FLCN Mutation p.R477*

\section{Case Presentation}

Disease History

Female patient S., 26 years of age, visited a urologist at the Research and Counseling Division of N.N. Blokhin National Medical Research Center of Oncology in 2020 to verify the diagnosis and obtain advice on follow-up and prognosis. The disease manifested in 2016 when a mass lesion of the right kidney was detected in another clinic by ultrasonography and later confirmed by abdominal computed tomography (CT). Laparoscopic resection of the right kidney was performed on September 2016. A pathomorphological report described clear cell RCC (G1) within the renal parenchyma of the right kidney. Spontaneous pneumothorax on July 2016 was noted in the disease history. In March 2020, a mass lesion of the left kidney was detected by abdominal magnetic resonance imaging (MRI) at a scheduled visit to the clinic. The patient was hospitalized to undergo testing and surgery.

\section{Laboratory and Instrumental Testing}

Dynamic renal scintigraphy showed that perfusion was preserved in both kidneys (glomerular filtration rates of $66 \mathrm{~mL} / \mathrm{min}$ on the right and $61.8 \mathrm{~mL} / \mathrm{min}$ on the left). Abdominal and pelvic spiral CT (SCT) showed tumor of the left kidney, $32 \times 30 \mathrm{~mm}$, and diffuse changes in the liver parenchyma. Chest spiral CT exhibited air cysts in both lungs (Fig. 1).

\section{Treatment and Pathomorphological Diagnosis}

Surgery involving left lumbotomy and resection of the left kidney with tumor nodes was performed on March 16, 2020. A pathomorphological examination was conducted on March 2020 and showed chromophobe RCC (Fig. 2) of 15, 10, and $3 \mathrm{~mm}$ in the largest dimension, limited to the kidney tissue. Tumor growth was not detected at resection margins. The main diagnosis was right RCC, multifocal left RCC, pT1N0M0, stage I. The concomitant diseases were right spontaneous pneumothorax (condition after thoracoscopy and right pleural cavity drain), distal catarrhal reflux esophagitis, and chronic gastritis.

\section{Molecular Genetic Analysis}

Blood was collected in a vacuum tube with EDTA. Genomic DNA was isolated from the blood using a DNA-sorb-B kit (Interlabservice, Russia). To test for somatic mutations, DNA was isolated from $310-\mu \mathrm{m}$ sections of a paraffin block with a chromophobe carcinoma fragment. The block was selected as having a higher carcinoma cell content by pathomorphology. DNA isolation from the paraffin block sections was conducted using a QIAamp DNA FFPE Tissue Kit (Qiagen, Hilden, Germany). The DNA concentration was measured using a Qubit 3.0 fluorometer (Thermo Fisher Scientific, Waltham, MA, USA). Polymerase chain reaction to amplify protein-coding FLCN exons 4-14 was conducted with blood DNA on a C1000 thermal cycler (Bio-Rad, Hercules, CA, USA). The primers and reaction conditions were applied based on a published protocol [8]. Sanger sequencing of the polymerase chain reaction products was performed with a BigDye Terminator version 3.1 Cycle Sequencing Kit and 3,500 genetic analyzer (Thermo Fisher Scientific). Annotation of the germline mutation detected was performed using the HGMD, ClinVar, and COSMIC databases as recommended [9]. The heterozygous nonsense mutation c.1429C $>$ T (p.R477*) was detected (Fig. 3a). The mutation (dbSNP: rs879255678, ClinVar: 253251) is classed with pathogenic mutations (FATHMM score 0.93, HGMD score 0.99, MAF < 0.01) and has been characterized in carcinogenesis (COSM6925131) and described as a cause of BHDS (HGMD CM051477). This finding confirmed the diagnosis of BHDS in the patient.

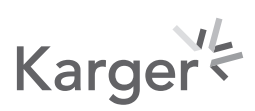



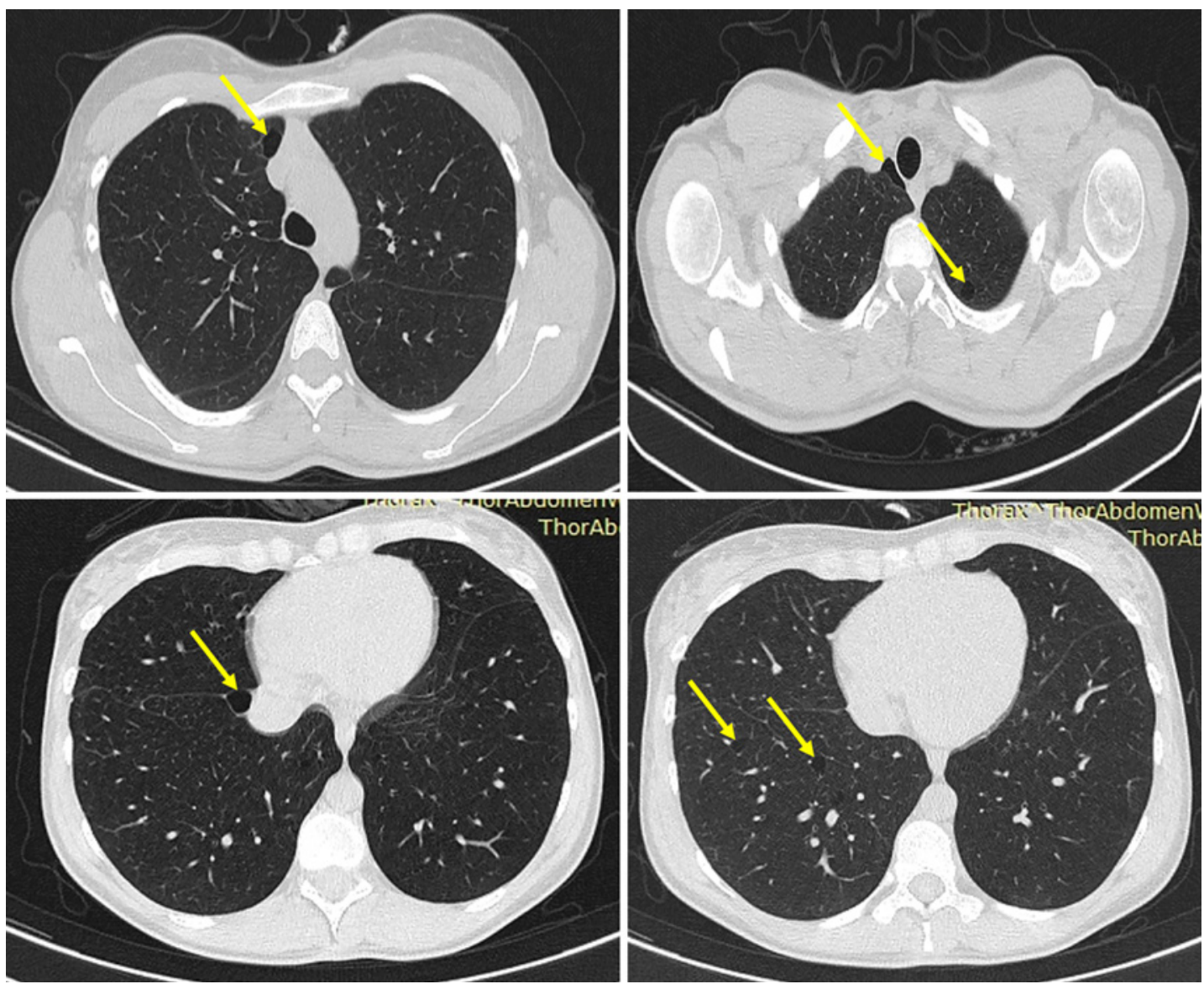

Fig. 1. Multiple air cysts (indicated with arrows) in the lungs of patient S.

A library of amplified short tumor DNA fragments was obtained using the Ion AmpliSeq Comprehensive Cancer Panel according to the Thermo Fisher Scientific protocols; semiconductor sequencing was conducted using an Ion S5 instrument. The mean amplicon coverage was 593 reads, sufficient for analyzing the somatic mutation profile. Sequencing results were analyzed using Torrent Suite software, including base caller to perform a primary analysis, Torrent Mapping Alignment Program to align the reads on a reference genome (NCBI build 37/ hg19), and Variant Caller to identify the nucleotide sequence variants. The clinical significance of genetic variants was evaluated using the ClinVar and COSMIC databases. To detect possible allelic imbalance, the BAM file from new-generation sequencing (NGS) was analyzed using the OncoCNV program [10]. The observed set of somatic mutations included 4 variants of uncertain significance; known cancer driver mutations were not detected (Table 1). A second inactivating mutation, c.58_59delfs, was found in FLCN exon 4 and confirmed by Sanger sequencing (which additionally confirmed that tumor DNA carried the germline FLCN mutation detected in blood DNA). Based on the OncoCNV analysis, major chromosome aberrations in the tumor included deletions from chromosomes 7, 16, 19, and 21; loss of the long arm of the X chromosome; and TP53 deletion (Fig. 3b). Note additionally that variants of uncertain significance of one allele and deletion of the other allele were observed in the NF2 tumor suppressor gene.

\section{Genetic Counseling}

The patient was referred to a medical-genetic counseling in view of the young age at disease onset and metachronous bilateral tumors of the kidneys. The patient did not have a 


\section{Case Reports in Oncology}

\begin{tabular}{l|l}
\hline Case Rep Oncol 2021;14:963-971 \\
\hline DOI: 10.1159/000516763 & $\begin{array}{l}\odot \text { 2021 The Author(s). Published by S. Karger AG, Basel } \\
\text { www.karger.com/cro }\end{array}$ \\
\hline
\end{tabular}

Mikhaylenko et al.: Case of Birt-Hogg-Dube Syndrome with the FLCN Mutation p.R477*

Fig. 2. Chromophobe RCC, hematoxylin-eosin staining, magnification $\times 200$. RCC, renal cell cancer.
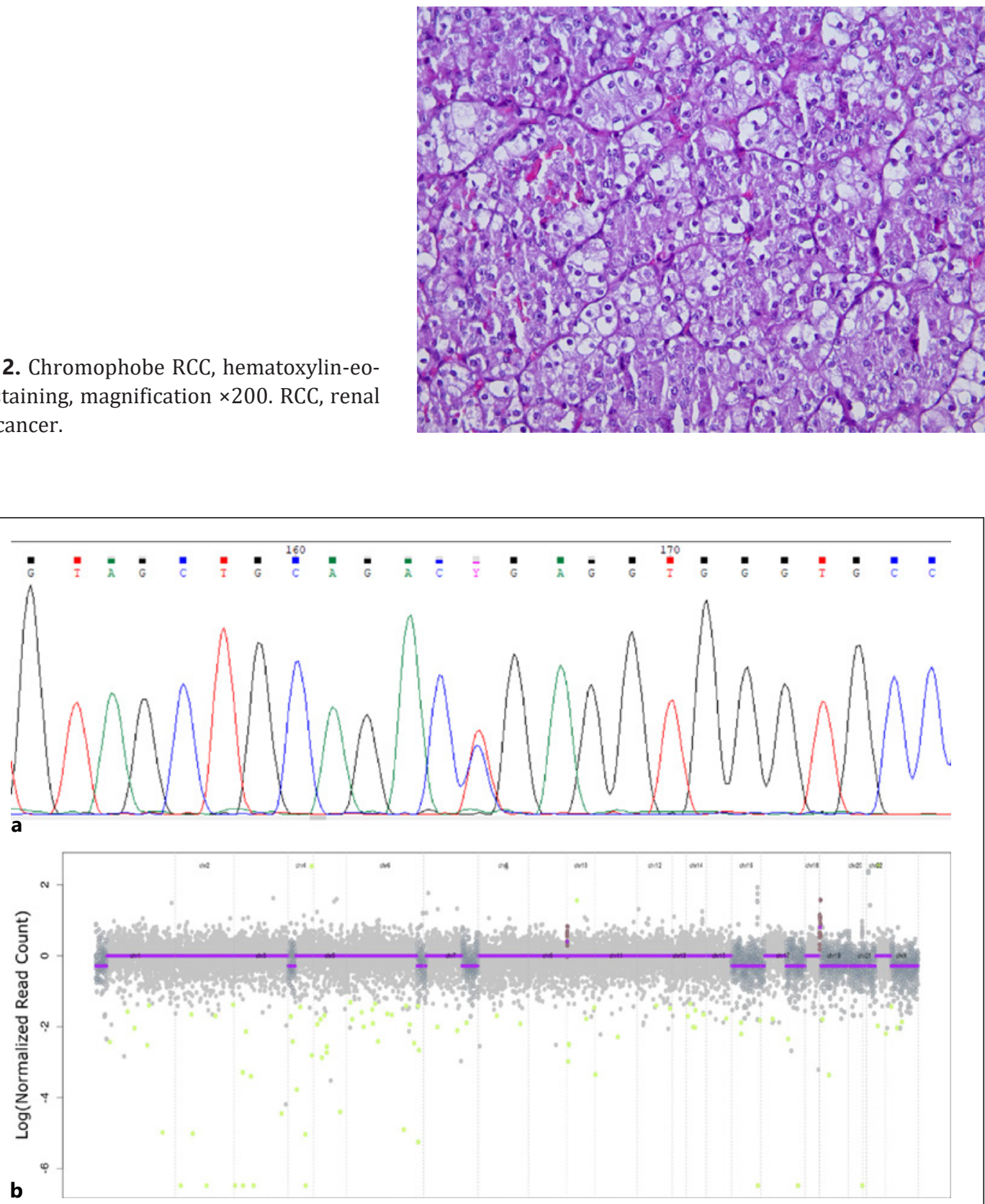

Fig. 3. Molecular genetic analysis. Sequencing of the c.1429C $>\mathrm{T}\left(\mathrm{p} . \mathrm{R} 477^{*}\right)$ mutation, the substitution C $>\mathrm{T}$ in exon 12 is indicated with Y (a); allelic imbalance in the tumor based on the OncoCNV analysis (b).

family history of cancer. Data on radiography and CT of the lungs were unavailable for both parents. No skin lesions were detected by visual assessment. Given the patient's young age, bilateral renal tumors of different histological types (including chromophobe carcinoma), and history of spontaneous pneumothorax, medical-genetic testing for FLCN mutations was recommended to verify the BHDS diagnosis. Based on the total set of laboratory and clinical findings, Patient $\mathrm{S}$. was advised to undergo a dermatological examination with dermatoscopy every 6-12 months, lung CT prior to a long air flight or scheduled general anesthesia, abdominal and pelvic MRI/contrast CT every 2-3 years, kidney ultrasound annually, thyroid 
Table 1. Somatic mutations found in the chromophobe renal cell carcinoma of the patient

\begin{tabular}{|c|c|c|c|}
\hline Chromosome & Gene & Mutation & Class \\
\hline 2 & NCOA1 & c.1816A>G (p.K606E) & VUS \\
\hline 7 & AKAP9 & c.4127G>C (p.S1376T) & Likely benign \\
\hline 8 & NCOA2 & c.3635G>A (p.G1212D) & VUS \\
\hline 9 & FANCC & c.29G>A (p.C10Y) & Likely benign \\
\hline 12 & KMT2D & c.11219_11220insGCA (p.3740_3741insQ) & Benign \\
\hline 15 & $B L M$ & c.1807G>C (p.A603P) & VUS \\
\hline 17 & FLCN & c.58_59del (p.F20fs) & Likely pathogenic \\
\hline 22 & $N F 2$ & c.G1004A (p.R335H) & VUS \\
\hline
\end{tabular}

VUS, variant of uncertain significance.

and parathyroid ultrasonography annually, and colonoscopy after 40 years of age. The risk of BHDS transmission to the offspring is $50 \%$ according to the autosomal dominant mode of inheritance. Given the incomplete penetrance and variation in age at disease manifestation, one of the patient's parents might carry the mutation. Therefore, molecular genetic testing for the c.1429C>T (p.R477*) mutation was recommended for the patient's first-degree relatives.

\section{Discussion}

Our molecular genetic testing confirmed BHDS, indicating that Patient S. is still at a higher risk of developing spontaneous pneumothorax and RCC in the remaining renal parenchyma. Based on the guidelines for managing patients with hereditary RCC, we believe that surgical resection of multiple tumors was a reasonable choice in this case. For example, intense follow-up without resection of renal tumors until they reach $3 \mathrm{~cm}$ in diameter is used to manage patients with von Hippel-Lindau syndrome, which is the best-studied form of hereditary RCC. A similar approach is possible for patients with BHDS or type 1 hereditary papillary RCC. However, this approach is unsuitable for tumors with a higher malignancy grade in other hereditary RCC forms, such as papillary RCC type 2 in hereditary leiomyomatosis and RCC, where the tactics of surgical treatment are similar to those in sporadic RCC [2].

Skin neoplasms were found on the head, neck, and upper trunk in patients with BHDS but were not detected in the proband. Cutaneous fibrofolliculomas are the most common sign of BHDS, but tumors usually do not develop in patients with BHDS until their middle age. Lung cysts are the main BHDS-associated pathologies in young patients with BHDS. Cysts start to form as early as adolescence, and $30 \%$ of patients with BHDS have a history of spontaneous pneumothorax by 40 years of age. Because of the high risk of life-threatening pneumothorax in young carriers of germline FLCN mutations, for example, during intense exercise even when lacking overt signs of the disease and decrease in external respiration, diagnosing BHDS in a timely manner is therefore greatly important $[3,11]$. Patients with suspected BHDS are advised to undergo molecular genetic testing as soon as they reach 20 years of age. When a germline FLCN mutation is detected and the diagnosis is confirmed, MRI is recommended every 3 years. If a renal tumor is detected, MRI or low-dose CT should be repeated annually to check the target organs for pathological signs [1].

\section{Karger'}


The germline p.R477* FLCN mutation was first identified in a patient who had spontaneous pneumothorax as the first BHDS manifestation without objective signs to suspect renal neoplasia. Schmidt et al. [12] reported the findings with a description of 51 families with BHDS (case no. 7707). Note that 4 of the 5 nonsense mutations observed in different families affect exon 12. Three of the mutations produce a stop codon at position 463 , and one is the substitution p.R477* [12]. More recently, the p.R477* mutation was observed in a 64-year-old female Korean patient who had no renal tumor; lung cysts and pneumothorax events were the most severe signs of BHDS in the patient and her daughter [13]. Another case has been described in Spain, where the germline c.1429C>T (p.R477*) mutation was detected in a 49-year-old male patient with multiple fibrofolliculomas [14]. Thus, patients with fibrofolliculomas and lung cysts have been described to carry the p.R477* mutation in the available literature. Our Patient $S$. is the first case of renal tumors in BHDS due to the p.R477* mutation. This fact emphasizes again that the mutation varies in expressivity and that BHDS is heterogeneous among different patients. It is of interest to note that the c. $1429 \mathrm{C}>\mathrm{T}$ (p.R477*) mutation is only $3 \mathrm{bp}$ away from the end of exon 12 and may distort the FLCN mRNA splicing in addition to generating a premature stop codon according to a bioinformatics study [15].

Activation of mTOR kinase is thought to be the key pathogenetic event that leads to carcinogenesis at the molecular level in BHDS. It has consequently been assumed that mTOR inhibitors may be effective in BHDS-associated tumors, by analogy with therapy for tuberous sclerosis. This assumption has not received sufficient support from clinical studies [3]. Unfortunately, oncogene-activating driver mutations or 2 inactivating mutations in a known tumor suppressor gene other than FLCN were not detected in our NGS-based analysis of point mutations and OncoCNV analysis of aberrations. Therefore, additional off-label drugs, as part of the tumor-agnostic approach to treating cancer with chemotherapeutics and targeted agents, could not be recommended for use in Patient $S$. in case of disease progression at the given assessment stage. Patient S. is further managed as a localized non-clear cell RCC case according to the abovementioned protocol.

\section{Conclusions}

In this way, the p.R477* mutation has been described by other authors in patients with fibrofolliculomas and lung cysts, but not in those with RCC, while RCC was the first manifestation of BHDS in our case. The case report may help geneticists, oncologists, and other specialists to better understand the clinical and genetic heterogeneity of BHDS in various populations.

\section{Acknowledgements}

The authors thank the Facility Department, Research Centre for Medical Genetics, for the opportunity to use Ion S5 sequencer during this study.

\section{Statement of Ethics}

Written informed consent was obtained from the patient for publication of this case report and any accompanying images. This case report was approved by the local Ethics Committee at Research Centre for Medical Genetics.

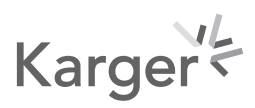




\section{Case Reports in Oncology}

Case Rep Oncol 2021;14:963-971

\begin{tabular}{l|l}
\hline DOI: 10.1159/000516763 & (c)21 The Author(s). Published by S. Karger AG, Basel
\end{tabular} www.karger.com/cro

Mikhaylenko et al.: Case of Birt-Hogg-Dube Syndrome with the FLCN Mutation p.R477*

\section{Conflict of Interest Statement}

The authors have no conflicts of interest to declare.

\section{Funding Sources}

This case study was supported by the Ministry of Science and Higher Education of the Russian Federation (Grant No. 01201363638).

\section{Author Contributions}

D.S.M. conducted the molecular genetic diagnosis of BHDS, detected the germline mutation, and wrote the majority of sections for the article; V.B.M., A.V.K., and S.D.N. performed the instrumental and clinical laboratory tests and surgery; M.G.F. collected biological material and performed the medical genetic counseling for the proband; K.I.A. conducted the NGS-based analysis of somatic mutations; N.A.K. pathomorphologically examined the left kidney tumors of the patient; A.V.S. collected the family history and assessed the patient; A.S.T. conducted the bioinformatics analysis of somatic mutations with NGS data; M.V.N. participated in writing the introduction and discussion of the manuscript; D.V.Z. prepared the illustrations and formatted the manuscript. All the authors have read and approved the manuscript.

\section{Availability of Data and Material}

The Sanger sequencing chromatogram of the exon 12 FLCN with forward primer and CCP raw sequencing reads of the tumor sample can be found in the Laboratory of Epigenetics website (http://www.epigenetic.ru/projects/renal-cancer/bhd1213f_G12_07.ab1) and NCBI BioProject repository (https://www.ncbi.nlm.nih.gov/bioproject/PRJNA699048), correspondingly.

\section{References}

1 Gaur S, Turkbey B, Choyke P. Hereditary renal tumor syndromes: update on diagnosis and management. Semin Ultrasound CT MR. 2017;38(1):59-71.

2 Maher ER. Hereditary renal cell carcinoma syndromes: diagnosis, surveillance and management. World J Urol. 2018;36(12):1891-8.

3 Steinlein OK, Ertl-Wagner B, Ruzicka T, Sattler EC. Birt-Hogg-Dube syndrome: an underdiagnosed genetic tumor syndrome. J Dtsch Dermatol Ges. 2018;16(3):278-83.

4 Nickerson ML, Warren MB, Toro JR, Matrosova V, Glenn G, Turner ML, et al. Mutations in a novel gene lead to kidney tumors, lung wall defects, and benign tumors of the hair follicle in patients with the Birt-Hogg-Dubé syndrome. Cancer Cell. 2002;2(2):157-64.

5 Schmidt LS, Linehan WM. FLCN: the causative gene for Birt-Hogg-Dube syndrome. Gene. 2018;640:28-42.

6 Schmidt LS, Linehan WM. Clinical features, genetics and potential therapeutic approaches for Birt-Hogg-Dubé syndrome. Expert Opin Orphan Drugs. 2015;3(1):15-29.

7 Furuya M, Hasumi H, Yao M, Nagashima Y. Birt-Hogg-Dube syndrome-associated renal cell carcinoma: histopathological features and diagnostic conundrum. Cancer Sci. 2020;111(1):15-22.

8 Sattler EC, Reithmair M, Steinlein OK. Kidney cancer characteristics and genotype-phenotype-correlations in Birt-Hogg-Dubé syndrome. PLoS One. 2018;13(12):e0209504.

9 Li MM, Datto M, Duncavage EJ, Kulkarni S, Lindeman NI, Roy S, et al. Standards and guidelines for the interpretation and reporting of sequence variants in cancer: a joint consensus recommendation of the Association for Molecular Pathology, American Society of Clinical Oncology, and College of American pathologists. J Mol Diagn. 2017;19(1):4-23. 
10 Boeva V, Popova T, Lienard M, Toffoli S, Kamal M, Le Tourneau C, et al. Multi-factor data normalization enables the detection of copy number aberrations in amplicon sequencing data. Bioinformatics. 2014;30(24):344350 .

11 Liu Y, Xing H, Huang Y, Meng S, Wang J. Familial spontaneous pneumothorax: importance of screening for BirtHogg-Dube syndrome. Eur J Cardiothorac Surg. 2020;57(1):39-45.

12 Schmidt LS, Nickerson ML, Warren MB, Glenn GM, Toro JR, Merino MJ, et al. Germline BHD-mutation spectrum and phenotype analysis of a large cohort of families with Birt-Hogg-Dubé syndrome. Am J Hum Genet. 2005; 76(6):1023-33.

13 Lee JH, Jeon MJ, Song JS, Chae EJ, Choi JH, Kim GH, et al. Birt-Hogg-Dube syndrome in Korean: clinicoradiologic features and long term follow-up. Korean J Intern Med. 2019;34(4):830-40.

14 Fuertes I, Mascaro-Galy JM, Ferrando J. Birt-Hogg-Dube syndrome in a patient with cutaneous symptoms and a c.1429C>T;p.R477X mutation in exon 12 of the folliculin gene. Actas Dermosifiliogr. 2009;100(3):227-30.

15 Xiong HY, Alipanahi B, Lee LJ, Bretschneider H, Merico D, Yuen RK, et al. RNA splicing. The human splicing code reveals new insights into the genetic determinants of disease. Science. 2015;347(6218):1254806. 